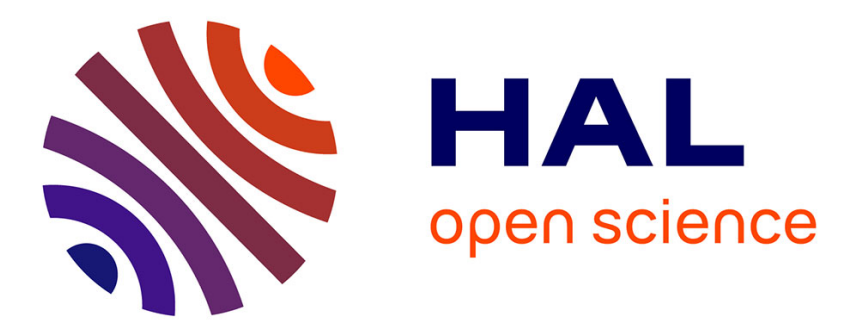

\title{
Information et consentement du patient dans le cadre de l'urgence en masso-kinésithérapie
}

François Vialla

\section{To cite this version:}

François Vialla. Information et consentement du patient dans le cadre de l'urgence en massokinésithérapie. L'urgence en kinésithérapie: Risques et Enjeux pour la profession, Faculté de droit Université Toulouse I Capitole - Faculté libre de droit de l'institut Catholique de Toulouse - Association Santéjuris, Nov 2018, Toulouse, France. hal-02318131

\section{HAL Id: hal-02318131 \\ https://hal.umontpellier.fr/hal-02318131}

Submitted on 26 Oct 2021

HAL is a multi-disciplinary open access archive for the deposit and dissemination of scientific research documents, whether they are published or not. The documents may come from teaching and research institutions in France or abroad, or from public or private research centers.
L'archive ouverte pluridisciplinaire HAL, est destinée au dépôt et à la diffusion de documents scientifiques de niveau recherche, publiés ou non, émanant des établissements d'enseignement et de recherche français ou étrangers, des laboratoires publics ou privés.

\section{다)(1) $(5$}

Distributed under a Creative Commons Attribution - NonCommerciall 4.0 International 


\section{Note de la Rédaction}

Les actes du colloque « L'urgence en kinésithérapie : Risques et Enjeux pour la profession », organisé par la Faculté libre de droit de l'institut Catholique de Toulouse, la Faculté de droit de l'Université Toulouse I Capitole et l'association Santéjuris, à Toulouse le 20 octobre 2018 sont copubliés en partenariat au sein de "Kinésithérapie, la Revue », éditée par Elsevier SAS, et «Lexbase »SA.

- Monnoyer MC. Introduction au colloque «L'urgence en kinésithérapie : Risques et Enjeux pour la profession ». Kinesither Rev 2019;19(210).

- Jean S. Propos introductifs relatifs à l'urgence en kinésithérapie. Kinesither Rev 2019;19(210).

- Mangematin C. La responsabilité civile du masseur-kinésithérapeute dans le cadre de l'urgence de l'article L.4321-1 du Code de la Santé Publique. Kinesither Rev 2019;19(210).

- Cazalbou P. De la responsabilité pénale du masseur-kinésithérapeute face à l'urgence. Kinesither Rev 2019;19(210).

- Rocton R. L'urgence en kinésithérapie, point de vue de l'expert. Kinesither Rev 2019;19(210).

- Vialla F. Information et consentement du patient dans le cadre de l'urgence en massokinésithérapie. Kinesither Rev 2019;19(210).

- Gedda M. Diagnostics croisés du médecin-prescripteur et du masseur-kinésithérapeute en contexte d'urgence. Kinesither Rev 2019;19(210).

Ces communications ont préalablement été validées par les organisateurs, selon une procédure qui leur appartient. En ce sens, Kinésithérapie la Revue n'a pas effectué de contrôle éditorial de ces contenus qui respectent les règles d'éthique et de déontologie.'

\section{Information et consentement du patient dans le cadre de l'urgence en masso-kinésithérapie}

Information and consent of the patient in the context of physiotherapy emergency

\section{François Vialla}

Professeur des universités

Directeur du Centre Européen d'Etudes et de Recherche Droit \& Santé

UMR5815 université de Montpellier

39, rue de l'Université

34060 MONTPELLIER

f.vialla@gmail.com

Conflit d'intérêt :

L'auteur déclare n'avoir aucun conflit d'intérêt en lien avec le présent article. 
Résumé :

Les dispositions de l'article L4321-1 précisent qu' «en cas d'urgence et en l'absence d'un médecin, le masseur-kinésithérapeute est habilité à accomplir les premiers actes de soins nécessaires en masso-kinésithérapie ». La mise en œuvre de ce nouveau dispositif légal soulève de nombreuses interrogations au nombre desquelles figurent les questions de l'information due au patient et du recueil de son consentement. L'urgence peut-elle être source d'une exception au respect de ces droits incontournables de la personne. Afin de mieux cerner les enjeux et logiques des problématiques liées à une éventuelle dispense, il convient de s'interroger sur les fondements juridiques des obligations d'information et de respect de la volonté du sujet.

\begin{abstract}
:
The provisions of the L4321-1 article from the public health Code specify that 'in case of emergency and in the absence of a medical doctor, the physiotherapist is entitled to perform the first acts of necessary care in physiotherapy. The implementation of this new legal system raises many questions, including the matter of patient's information and needful collection of his consent. Does the urgency can be an exception to the respect of these essential rights of the person. In order to better understand the stakes and the consistencies of the issues related to a possible exemption, it seems necessary to examine the legal bases of both obligations of information and respect for the will of the subject.
\end{abstract}

Mots clés : Autonomie de la volonté ; Consentement libre et éclairé ; Droit des patients ; Droits fondamentaux ; Information ; Respect de la volonté

Keywords: Autonomy of the will; Free and informed consent; Patient's right; Fundamental rights; Information; Respect for the will 
La question abordée, «Information et consentement du patient dans le cadre de l'urgence en massokinésithérapie », mérite indéniablement une analyse, qui ne saurait s’inscrire exclusivement dans le champ juridique. Que fait ici le droit ? On pourrait, paraphrasant Talleyrand au congrès de Vienne, répondre : «Il fait que vous y êtes ${ }^{1}$.

Mais le juriste s'empresse de rappeler que «Toutes les normes qui gouvernent la vie des hommes en société, dans une société globale donnée, ne sont pas toutes des règles de droit»[1]. La normativité applicable aux questionnements posés par l'information et le consentement ne saurait être cantonnée à la seule analyse juridique. Celle-ci, cependant, demeure nécessaire afin que les praticiens puissent, aussi sereinement que possible, envisager les obligations et devoirs pesant sur eux en la matière.

Le droit, entendu comme mécanisme d'encadrement du fait social, ne peut être abordé sans que l'on s'intéresse de prime abord aux circonstances de son intervention. Les textes doivent, en effet, être analysés dans la perception du contexte.

Il ne nous incombe pas dans le cadre présent de nous étendre sur la notion d'urgence ${ }^{2}$, d'autres, plus qualifiés et plus compétents, assument cette lourde et délicate tâche. Pour autant on peut se permettre de souligner que l'absence d'approche univoque peut conduire à l'équivoque, voire à la controverse [2-4].

La confrontation du contexte et des textes, du fait social et du droit, conduit à se poser cette question qui peut paraître provocante ou simplement naïve: existe-t-il une urgence en kinésithérapie? On pourrait être tenté de répondre en se défaussant: «l'urgence en massokinésithérapie doit bien exister puisqu'elle existe... dans la loi »!

L'article L4321-1 du Code de la santé publique, en effet, dispose in fine qu' «En cas d'urgence et en l'absence d'un médecin, le masseur-kinésithérapeute est habilité à accomplir les premiers actes de soins nécessaires en masso-kinésithérapie. Un compte rendu des actes accomplis dans ces conditions est remis au médecin dès son intervention $»^{3}$.

Peut-on se contenter de noter la mention de l'urgence dans le dernier alinéa de l'article L4321-1 sans plus s'interroger sur ses contours, au risque de s'entendre dire comme le Vicomte de Valvert, sur un ton successivement Agressif, Amical, Descriptif, Curieux, Gracieux, Truculent, Prévenant, Tendre, Pédant, Cavalier, Emphatique, Dramatique, Admiratif, Lyrique, Naïf, Respectueux, Campagnard, Militaire, Pratique : «C'est un peu court jeune homme $»^{4}$ ?

\footnotetext{
${ }^{1}$ Talleyrand répondait à Hardenberg, plénipotentiaire de Prusse, qui demandait « Mais que fait ici le droit [public]) ?».

${ }^{2}$ Nous serons toutefois amenés à aborder la question sous l'angle de la dispense légale des obligations d'information et de recueil du consentement [voir infra II].

${ }^{3}$ CSP Article L4321-1 Modifié par Loi n²016-41 du 26 janvier 2016 - art. 123 Modifié par Loi n²016-41 du 26 janvier 2016 - art. 134.

${ }^{4}$ Rostand E. Cyrano de Bergerac, Acte 1 scène 4.
} 
Sans prétendre à l'exhaustivité de la réflexion, tentons de mieux approcher les éléments présents dans le texte. Le juriste «obsédé textuel» se livre souvent à une rigoureuse dissection terminologique. Chaque mot, chaque groupe de mots, ayant un sens précis, cette approche permet de mieux cerner les enjeux et logiques du cadre posé :

- «En cas d'urgence

- et en l'absence d'un médecin,

- le masseur-kinésithérapeute

- est habilité

- à accomplir

- les premiers actes de soins

- nécessaires

- en masso-kinésithérapie».

Au-delà de la condition d'urgence ${ }^{5}$ d'autres conditions sont posées par les dispositions légales. La première est relative à l'absence d'un médecin. Celle-ci, posée en terme très généraux, ne peut être interprétée comme l'absence du médecin du patient, du médecin traitant notamment. Cette notion d'absence peut être source de difficultés. S'agit-il d'une absence «physique » sur les lieux de la consultation où peut-on l'appréhender de manière plus large? L'appel au médecin régulateur, permet-elle de pallier la présence physique ? Plus largement une présence « distancielle ${ }^{6}$, prive-telle le masseur-kinésithérapeute de sa possibilité d'action?

Le texte se poursuit en prévoyant que le masseur-kinésithérapeute « est habilité à ».

Le terme habilité est ainsi défini par le Dictionnaire de l'Académie Française ${ }^{7}:$ «HABILITÉ $n . f$. XIV siècle. Emprunté du latin habilitas, au sens d'aptitude légale à [...] Droit : Aptitude, capacité légale à accomplir certains actes ou à exercer certains pouvoirs ».

Le texte consacre donc une « capacité juridique » à l'accomplissement d'actes.

Précisément, les actes dont il s'agit sont mentionnés comme étant «les premiers actes de soins nécessaires en masso-kinésithérapie ». L’habilitation est donc stricte, voire restrictive, seuls les actes initiaux entrant dans le champ de qualification de cette profession sont concernés et que, par ailleurs, la poursuite des soins peut n'être pas de la compétence du masseur-kinésithérapeute.

Ces premiers actes doivent en outre être «nécessaires », c'est-à-dire, selon le Dictionnaire de l'Académie Française ${ }^{7}$, ceux «Dont on ne peut se passer, dont on a absolument besoin pour quelque fin ». Le critère n'est pas celui de l'utilité mais est bien ancré dans l'indispensabilité.

\footnotetext{
${ }^{5}$ Voir Infra II.

${ }^{6}$ Rappelons que le préfixe $1 \tau \tilde{\eta} \lambda \varepsilon$, / têle, présent dans les termes télémédecine, télésanté, téléconsultation etc. signifie loin, au loin, à distance.

${ }^{7}$ https://academie.atilf.fr
} 
Il s'agit surtout d'actes de soins. Mais qu'entend-on par soin, que veut dire «soigner », qui plus est dans un contexte d'urgence?

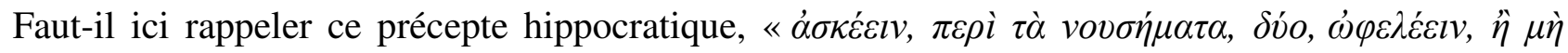

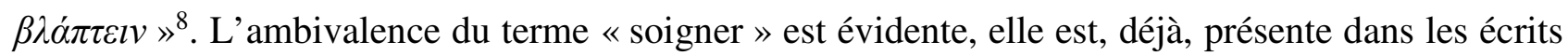
du médecin $\operatorname{Luc}^{9}$ [5] : «Un Samaritain, s'approcha, et banda ses plaies, en y versant de l'huile et du vin; [...] puis il [...] prit soin de lui $»^{10}$. L'acte de «traumatologie » (banda ses plaies), le savoirfaire, se double du souci du «prendre soin», du «savoir être ». Confiner le patient dans une « résidualité » liée à sa pathologie, son âge, son handicap aurait pour conséquence de transformer le praticien en un «technicien de l'organe malade»[6], ce qui serait la négation même de la qualification de «soignant ». Soigner est une aptitude plurielle qui relève d'abord d'une TÉ $\chi v \eta^{11}$,

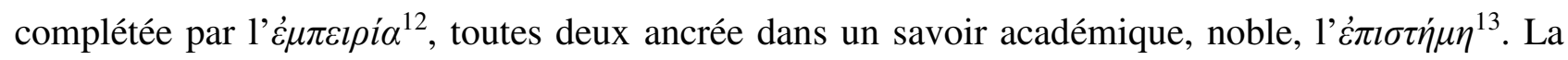
dimension technique, est incontournable, elle constitue le plus petit dénominateur commun attendu, Guy de Chauliac déjà écrivait dans la Cirugica magna ${ }^{14}$ : «En toute maladie, l'art commande la propre curation, excepté en trois cas, esquels suffit la curation large, perseruative et palliative. Le premier cas est quand le mal est simplement ou absolument incurable, comme ladrerie. Le second, quand le mal de soy est guerissable, mais il est vn patient desobéissant, ou qui ne peut souffrir, et soutenir la peine. Le troisième est quand la guarison de ce mal là engendreroit une pire maladie » [7].

Même en contexte d'urgence, un cadre juridique précis encadre l'acte de soin et, sauf exception légale, ce cadre doit être respecté.

Le Code civil dispose à l'article 16-3 : «Il ne peut être porté atteinte à l'intégrité du corps humain qu'en cas de nécessité médicale pour la personne ou à titre exceptionnel dans l'intérêt thérapeutique d'autrui. / Le consentement de l'intéressé doit être recueilli préalablement hors le cas où son état rend nécessaire une intervention thérapeutique à laquelle il n'est pas à même de consentir ».

L'autorité, scientifique et technique, du soignant, fondée sur son savoir, est contrebalancée par le pouvoir reconnu au patient. L'expression de la volonté de la personne, son consentement ${ }^{15}$, est nécessaire à la licéité de l'acte envisagé.

\footnotetext{
${ }^{8}$ Hippocrate (Epidémies) : «Avoir, dans les maladies, deux choses en vue : être utile ou du moins ne pas nuire »; «Primum non nocere, deinde curare».

${ }^{9}$ L'évangéliste Luc était médecin comme cela peut être compris à la lecture de l'Epitre de Paul aux Colossiens 4,14.

${ }^{10}$ Luc 10-29/35.

11 Tekhnè.

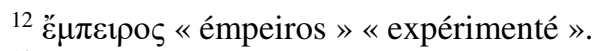

${ }^{13}$ Epistêmê.

14 Traité en 7 livres, écrit en Avignon en 1363, éd. princeps, Venise, 1498 ; https://gallica.bnf.fr/ark:/12148/bpt6k9656319n?rk=214593;2

${ }^{15}$ Code civil Art. 16-3, al.2 ; Code de la santé publique Art. L1111-4.
} 
Savoir et savoir-faire légitiment l'action du praticien pour autant que soient respectés des critères objectifs et subjectifs qui l'encadrent. L'ambivalence avérée de la prestation de soin met à la charge du praticien non seulement des obligations de science et de technique, mais encore des obligations de conscience et d'humanisme [8].

Dans son rapport annuel pour 2010, la Cour de cassation soulignait que «Le médecin [il en va de même pour le masseur-kinésithérapeute] est tenu d'une obligation de science et de conscience, ce que conforte la prise en compte réaffirmée récemment de ses obligations déontologiques [...] Sa responsabilité peut être engagée non seulement en cas de faute technique, mais encore en cas de faute d'humanisme, laquelle trouve sa traduction fréquente dans le manquement à l'obligation d'information, et au droit qu'a le patient à consentir, de manière éclairée, à l'acte médical » ${ }^{16}$. Objectivement considéré, l'acte doit être ancré dans la «nécessité médicale pour la personne ${ }^{17}$, dans la proportionnalité «risques encourus /bénéfice escompté ${ }^{18}$, dans le respect des règles de Arte, des «connaissances médicales avérées ${ }^{19}$ et, pour le masseur-kinésithérapeute, dans les «données actuelles de la science $»^{20}$. Subjectivement appréhendé, l'acte nécessite que le soignant dispense une information ${ }^{21}$ et veille à sa compréhension ${ }^{22}$, qu'il prodigue des conseils ${ }^{22}$, émette des préconisations ${ }^{23}$ et, enfin, qu'il recueille et respecte le consentement du patient ${ }^{24}$.

On le voit les critères juridiques de licéité de la prestation de soin sont précis. Au nombre des conditions émises par les textes, l'information ${ }^{25}$, le recueil et le respect du consentement ${ }^{26}$ paraissent incontournables.

Il convient de mieux en cerner les enjeux et logiques (I) afin de dessiner les contours des devoirs du soignant à cet égard, notamment en contexte d'urgence (II).

Si le consentement, précédé d'une information, constitue une condition nécessaire, mais non suffisante, à la validité de tout acte de soin, se pose la question de la possible exception à ces devoirs en cas d'urgence constatée ${ }^{27}$.

16

https://www.courdecassation.fr/publications_26/rapport_annuel 36/rapport 2010_3866/etude_droit_3872/e_droit 3873 /obligation_information_3874/obligation_information_medicale_19400.html

${ }^{17}$ Code civil Art. A6-3, al.1.

${ }^{18}$ CSP Art. L1110-5.

${ }^{19}$ CSP Art. L1110-5.

${ }^{20}$ Le Code de déontologie de la profession de masseur-kinésithérapeute se réfère, en effet, aux « données actuelles de la science » (Article R4321-80 Décret n²008-1135 du 3 novembre 2008 - art. 1) ; Les Codes de déontologie des médecins (CSP Art. R4127-32), des chirurgiens-dentistes (CSP Art. R4127-233) et des infirmiers (CSP Art. R4312-10) usent de la formule «Données acquises de la science » et le Code de déontologie des sages-femmes (Art. R4127-325) utilise la notion de «Données scientifiques du moment».

${ }^{21}$ CSP Art. L1111-2 ; CSP Art. R4321-83.

${ }^{22}$ CSP Art. R4321-83.

${ }^{23}$ CSP Art. L1111-4, al.1.

${ }^{24}$ CSP Art. L1111-4 ; CSP Art. R4321-84.

${ }^{25}$ CSP Art. L1111-2.

${ }^{26}$ CSP Art. L1111-4.

${ }^{27}$ S'agissant de l'information l'article L1111-2, al.2 du CSP dispose : «Seules l'urgence ou l'impossibilité d'informer peuvent l'en dispenser ». 


\section{I/ Les fondements de l'information et du recueil du consentement}

Les obligations légales et réglementaires, informer, recueillir et respecter le consentement, ne sont pas de simples procédures formelles, elles reflètent l'esprit de la Loi : faire du patient un acteur, un sujet des soins et non le circonscrire à l'état d'objet du soin.

On se doit, ici, de relever une différence majeure entre les situations respectives des praticiens et du patient s'agissant de la décision de soin. Les premiers sont indéniablement concernés par la décision [9] à prendre, seul le second est impliqué par la décision prise.

Pour être plus compréhensible on nous pardonnera d'user d'une métaphore. La relation de soin est semblable à une... omelette au lard! Dans l'omelette au lard, deux animaux interviennent, mais si la poule [le soignant] est simplement concernée par la recette, le porc [le patient] lui est impliqué dans le plat! Plus académiquement, il convient de souligner que l'objet de la décision est distinct pour les deux parties, «dans la décision médicale [il en va de même en masso-kinésithérapie] prise à deux, il y a deux facettes distinctes car, si le médecin [il en va de même pour le masseurkinésithérapeute] est décidé, le malade doit se décider. La différence porte sur l'objet de la décision : pour le médecin [le masseur-kinésithérapeute], l'objet est un traitement pour le sujet pour le malade, l'objet c'est lui-même » [10].

Assurément la possibilité que le patient puisse comprendre l'information dispensée et qu'il soit en mesure d'émettre un consentement éclairé a longtemps été contestée. Ainsi le Pr. Portes soulignait que «Face au patient, inerte et passif, le médecin n'a en aucune façon le sentiment d'avoir à faire à un être libre, à un égal, à un pair qu'il puisse instruire véritablement. Tout patient est, et doit être pour lui, comme un enfant à apprivoiser, non certes à tromper - un enfant à consoler, non pas à abuser - un enfant à sauver, ou simplement à guérir, [...] le patient à aucun moment ne connaissant, au sens exact du terme, sa misère, ne peut vraiment consentir ni à ce qui lui est affirmé, ni à ce qui lui est proposé » [11].

On réalise le chemin parcouru en quelques décennies ! De devoir du soignant l'information est devenue un droit du patient et son consentement est érigé en condition nécessaire à la validité de l'acte : "l'information a été longtemps une prérogative exercée par un médecin qui appréciait en conscience si son patient devait en recevoir la délivrance. Le pouvoir du praticien résidait alors dans sa libre décision de donner ou non l'information. La reconnaissance, dans la loi nouvelle, d'un droit du malade à être informé, implique en contrepartie le pouvoir d'exiger $d u$ thérapeute la délivrance de l'information, celui de lui imposer une fois informé le choix de sa décision ${ }^{28}$, et enfin celui d'obtenir une indemnisation alors qu'aucune faute de technique médicale n'a été commise.

\footnotetext{
${ }^{28}$ Nous nous permettons de modérer cette affirmation, l'exigence d'information et de consentement du patient ne peut être entendu comme le droit de choisir et d'exiger un acte que le praticien ne préconiserait pas ; CE, ord. réf. 26 juillet
} 
L'autonomie du patient semble donc faire reculer le pouvoir du médecin, elle se manifeste à son tour comme une emprise exercée sur le praticien » [13].

L'évolution mise à jour reflète une véritable métamorphose de la relation de soin dans laquelle l'autonomie du patient est consacrée. Cette autonomie, cependant, ne doit pas devenir le «Cheval de Troie » des soignants qui, prétexte pris du pouvoir reconnu à la personne, ne sauraient négliger l'exigence d'accompagnement de la personne dans ses choix. On peut considérer que «la fiction juridique du droit des malades repose sur une égalité des statuts [...] (médecin et malade seraient des sujets de droit) mais qui ne peut feindre d'ignorer que l'égalité ne fait pas la similitude. Que le malade soit sujet de droit n'enlève pas la dépendance qui fait que, dans sa demande de soin, il est dépendant, vulnérable, fragilisé au point de ne pouvoir choisir seul, ou être laissé seul dans la solitude du choix, exalté au nom de l'autonomie. [...] La reconnaissance récente de l'autonomie de l'usager en droit de la santé n'annihile pas cette dépendance mais interroge la possibilité d'une autonomie dans la dépendance » [10].

La reconnaissance de l'autonomie de la volonté du patient n'est pas synonyme d'une neutralisation de la parole du soignant. Elle est plutôt le signe d'un humanisme recouvré et assumé dans la relation de soin.

Rappelons que le terme « humanisme » signifie «Doctrine, attitude philosophique, mouvement de pensée qui prend l'homme pour fin et valeur suprême, qui vise à l'épanouissement de la personne humaine et au respect de sa dignité ${ }^{29}$.

Comment ne pas noter la proximité de cette approche avec les dispositions de l'article 16 du Code civil : «La loi assure la primauté de la personne, interdit toute atteinte à la dignité de celle-ci et garantit le respect de l'être humain dès le commencement de sa vie $»^{30}$. On comprend dès lors que l'on puisse utiliser la notion de décision partagée entre le soignant et le patient, approche confirmée par l'analyse de l'alinéa premier de l'article L1111-4 du Code de la santé publique : «Toute personne prend, avec le professionnel de santé et compte tenu des informations et des préconisations qu'il lui fournit, les décisions concernant sa santé ». Loin de confiner le praticien dans un rôle passif et secondaire soumis au diktat du patient, le texte envisage bien que la décision est partagée, au sens de «prise avec ». Assurément les textes imposent la quête et le respect du consentement préalable de la personne ${ }^{31}:$ «Toute personne a le droit de refuser ou de ne pas recevoir un traitement. [...] Aucun acte médical ni aucun traitement ne peut être pratiqué sans le

$2017: \mathrm{n}^{\circ}$ 412618, D. et B. la haute juridiction relève qu'aucune disposition légale «ne consacrent, au profit du patient, un droit de choisir son traitement »; CE, 27 juillet $2018: \mathrm{n}^{\circ} 422241$ [12].

${ }^{29}$ Dictionnaire de l'Académie Française, https://academie.atilf.fr, $\mathrm{V}^{\circ}$ Humanisme.

${ }^{30} \mathrm{La}$ «structure » même du Code civil sous-tend cette dimension humaniste : Livre Ier : Des personnes ; Livre II : Des biens et des différentes modifications de la propriété ; L'être, le sujet, vient avant l'avoir, les choses.

${ }^{31}$ Code civil Art. 16-3 al.2 ; CSP Art. Art. R4321-84, al.1 : «Le consentement de la personne examinée ou soignée est recherché dans tous les cas. Lorsque le patient, en état d'exprimer sa volonté, refuse le traitement proposé, le masseur- 
consentement libre et éclairé de la personne et ce consentement peut être retiré à tout moment $»^{32}$. Le patient doit librement accepter l'acte préconisé, il peut tout aussi librement le refuser. Brassens ne chantait-il pas : "C'est même en revenant de chez cet antipathique / Qu'il tomba victime d'une indigestion critique / Et refusa le secours de la thérapeutique / Corne d'Aurochs/ Parce que c'était un Allemand, au gué, au gué / Qu’on devait le médicament, au gué, au gué » ${ }^{33}$.

Mais cela ne saurait conduire à considérer que le patient peut exiger du praticien qu'il réalise un acte que ce dernier ne préconise pas. Le Conseil d'Etat l'a rappelé dans deux décisions récentes ${ }^{34}$, la haute juridiction relève qu'aucune disposition légale «ne consacrent, au profit du patient, un droit de choisir son traitement ». On peut ajouter que le professionnel de santé, dès lors qu'il ne s'est pas confiné dans la neutralité et qu'il a émis des préconisations ${ }^{35}$, recouvre une réelle liberté ${ }^{36}$ et peut alors se retirer et refuser ses soins ${ }^{37}$.

L'évolution de la relation de soin fait donc du patient un égal du soignant, il ne s'agit bien évidemment pas de prétendre à une égalité de savoir, mais plutôt à une égalité de statut, patient et professionnel de santé sont des sujets de droit égaux ${ }^{38}$. Cette affirmation est essentielle pour cerner les fondements mêmes de l'obligation d'information qui incombe au praticien.

L'information permet, de première part, d'éclairer le consentement. Mais elle est bien davantage. Elle est aussi le signe du respect de la personne en tant que sujet en dépit de sa vulnérabilité. Les vers de Sophocle résonnent avec insistance : «C'est donc quand je ne suis plus rien, que je deviens

kinésithérapeute respecte ce refus après avoir informé le patient de ses conséquences et, avec l'accord de ce dernier, le médecin prescripteur $»$.

${ }^{32}$ CSP Art. L1111-4, al 1.

${ }^{33}$ Brassens, Corne d'Aurochs. Brassens se serait-il inspiré d'une déclaration d'Elsa Triolet : «Je ne pardonnerai jamais de devoir ma guérison aux singes d'outre-Atlantique»? V Ch. Jinker, Georges Brassens et Jacques Brel, Personal et social narratives in post-war chansons, Liverpool university press, 2005, Chap. 7, p.148-62, spécialement note 22.

${ }^{34} \mathrm{CE}$, ord. réf. 26 juillet $2017: \mathrm{n}^{\circ} 412618$, D. et $\mathrm{B}$. la haute juridiction relève qu'aucune disposition légale «ne consacrent, au profit du patient, un droit de choisir son traitement »; CE, 27 juillet $2018: \mathrm{n}^{\circ} 422241$ [12].

${ }^{35}$ CSP Art. L1111-4, al.1.

${ }^{36}$ CSP Art. R4321-59 : «Dans les limites fixées par la loi, le masseur-kinésithérapeute est libre de ses actes qui sont ceux qu'il estime les plus appropriés en la circonstance. Sans négliger son devoir d'accompagnement moral, il limite ses actes à ce qui est nécessaire à la qualité, à la sécurité et à l'efficacité des soins. Il agit de même pour ses prescriptions, conformément à l'article L. 4321-1. Il prend en compte les avantages, les inconvénients et les conséquences des différents choix possibles ».

${ }^{37}$ CSP Art. L1110-3, al. 7 : «Hors le cas d'urgence et celui où le professionnel de santé manquerait à ses devoirs d'humanité, le principe énoncé au premier alinéa du présent article ne fait pas obstacle à un refus de soins fondé sur une exigence personnelle ou professionnelle essentielle et déterminante de la qualité, de la sécurité ou de l'efficacité des soins. La continuité des soins doit être assurée quelles que soient les circonstances, dans les conditions prévues par l'article L. 6315-1 du présent code». Des limites à ce «refus de soigner» sont émise, l'urgence, le manquement à l'humanité, la continuité des soins ; Art. R4321-92: «La continuité des soins aux patients doit être assurée. Hors le cas d'urgence et celui où il manquerait à ses devoirs d'humanité, le masseur-kinésithérapeute a le droit de refuser ses soins pour des raisons professionnelles ou personnelles. S'il se dégage de sa mission, il en avertit alors le patient et transmet au masseur-kinésithérapeute désigné par celui-ci les informations utiles à la poursuite des soins ».

38 JO débats, Assemblée Nationale Compte rendu intégral, $2^{\mathrm{e}}$ séance du mardi 2 octobre 2001 15: Kouchner B., ministre délégué à la santé : "Il faut nous donner les moyens de sortir d'une relation trop souvent paternaliste. Il faut que les médecins admettent qu'ils ont en face d'eux des adultes, des partenaires ». 
vraiment un homme ${ }^{39}$. Instruire, informer permet de ne pas confiner le patient dans «sa pathologie », son âge ou son handicap. La jurisprudence à très tôt affirmé que l'information et le recueil du consentement étaient le signe du respect de la personne : «en violant cette obligation [d'obtenir le consentement du malade], imposée par le respect de la personne humaine, il [le chirurgien] commet une atteinte grave aux droits du malade, un manquement à ses devoirs proprement médicaux $[\ldots] \gg{ }^{40}$.

En 2001, la Cour de cassation affirmait, encore, «qu’un médecin ne peut être dispensé de son devoir d'information vis-à-vis de son patient, qui trouve son fondement dans l'exigence du respect du principe constitutionnel de sauvegarde de la dignité de la personne humaine, par le seul fait qu'un risque grave ne se réalise qu'exceptionnellement $»^{41}$. Comme le soulignait M. F. Chabas, «L'obligation médicale d'information ressortit à la protection de la dignité de la personne humaine. Elle est le corollaire, ou plutôt le préalable de l'obligation de recueillir le consentement du patient, obligation qui, sans elle n'aurait pas de sens. Quelle valeur aurait un consentement qui ne serait pas éclairé ? » [14].

Le recueil du consentement et son préalable, la dispensation de l'information, sont donc des obligations qui s'inscrivent donc dans une intensité normative forte, il s'agit, en effet, de respecter la dignité du patient !

Il convient de s'attacher à mieux cerner les exigences posées par le droit et, pour ce faire, de tenter de mieux définir les termes.

Informer est ainsi défini par le Dictionnaire de l'Académie Française : «2. Mettre au courant, instruire d'un fait, donner connaissance d'une nouvelle ${ }^{42}$. Le terme Informare signifie en latin : «mettre en forme, donner une forme; façonner, représenter, décrire », l'ancien français connaissait le verbe enformer ${ }^{43}$.

Il s'agit donc «d'instruire la personne », mais d'une manière particulière puisqu'il convient de mettre en forme un savoir afin de le rendre intelligible, compréhensible pour le « non sachant ».

Savatier remarquait à propos qu'entre le professionnel libéral et son client la relation fut d'abord celle «d'un profane à un initié » [15] avant de devenir «le rapport de l'ignorant au savant ${ }^{44}$. Il incombe, donc, au savant, de rendre sa science accessible à la compréhension de l'ignorant.

\footnotetext{
${ }^{39}$ Sophocle, Edipe à Colonne.

${ }^{40}$ Cass. req. 28 janvier 1942, Teyssier; Gaz. Pal, 62, 1942, 1, 177 ; D. 1942, 63 ; V. Les grandes décisions du droit médical LGDJ 2014, $2^{\mathrm{e}}$ Ed. Dir. Vialla F.

${ }^{41}$ Cass. 1re civ. 9 octobre 2001, no 00-14564; Bull. civ. I, no 249 ; D. 2001, juris. 3470, rapp. P. Sargos, note D. Thouvenin ; JCP éd. G 2002, II, 10045, note O. Cachard ; LPA, no 243, 6 déc. 2001, note C. Clément ; LPA no 52, 13 mars 2002, 17, note F. Marmoz; Resp. civ. et assur. 2001, comm. no 374 ; Les grandes décisions du droit médical LGDJ 2014, $2^{\mathrm{e}}$ Ed. Dir. Vialla F.

${ }^{42}$ Dictionnaire de l'Académie Française $9^{\mathrm{e}} \mathrm{V}^{\circ}$ informer Ed. https://academie.atilf.fr/9/

${ }^{43}$ Dictionnaire de l'Académie Française $9^{\mathrm{e}}$ Ed. https://academie.atilf.fr/9/

${ }^{44}$ Savatier, op.cit. p.191.
} 
Ainsi alerter le patient en lui assénant: «Vous souffrez d'une lésion traumatique d'un ou de plusieurs ligaments avec suspicion de rupture totale ou d'une avulsion de son enthèse ${ }^{45}$ ne constitue aucunement une information mais bien davantage une source de confusion anxiogène. Si, au contraire, je façonne mon savoir en annonçant à la personne qu'elle souffre d'une «entorse grave », mon discours est accessible à la compréhension. Un arrêt rendu en 1981 illustre bien cette nécessité de mise en forme du savoir du soignant : «[...] s'il est exact que le diagnostic indiqué par le docteur y... à sa cliente était celui de sinusite frontale double, et non celui de mucocele ${ }^{46}$, le chirurgien n'a employé l'expression de 'sinusite' que pour faire comprendre à la dame veuve x..., qui n'aurait pas eu l'intelligence du terme de 'mucocele', le genre d'affection dont elle était atteinte ; [...] [que la Cour d'appel] observe encore 'que le docteur y..., qui affirme avoir informé la dame x... de la gravite de son cas et des séquelles que l'opération pourrait entrainer, lui a, en parlant de sinusite frontale double, donné une information simple, approximative, intelligible et loyale, pour lui permettre de prendre la décision qu'il estimait s'imposer ${ }^{47}$.

On conçoit les réticences des professionnels quant à la capacité du patient à recevoir une information souvent complexe et, conséquemment, à consentir. Pour autant les textes sont clairs.

L'information et le recueil du consentement sont érigés en autant de droits ${ }^{48} \mathrm{du}$ patient et donc constituent des devoirs ${ }^{49}$ des soignants. La règle de droit ne fait, au demeurant, que traduire l'idée selon laquelle «Ce que vous faites pour moi, sans moi, vous le faites contre moi $»^{50}$.

S'ils posent des exigences, les textes précisent aussi les contours de ces droits.

Ainsi la Convention Européenne pour la protection des Droits de l'Homme et la dignité de l'être humain à l'égard des applications de la biologie et de la médecine (Convention sur les droits de l'homme et la biomédecine $)^{51}$ consacre son Chapitre II à la question du consentement. L'article 5 de la Convention pose une règle de principe : «Règle générale : Une intervention dans le domaine de la santé ne peut être effectuée qu'après que la personne concernée y a donné son consentement libre et éclairé. / Cette personne reçoit préalablement une information adéquate quant au but et à

\footnotetext{
${ }^{45} \mathrm{http}: / /$ dictionnaire.academie-medecine.fr/index.php?q=entorse $:$ torquere $:$ tordre $=>$ entorse.

${ }_{46}$ http://dictionnaire.academie-medecine.fr/index.php?q=mucocele : «Accumulation pathologique d'une substance mucoïde dans une cavité (mucocèle appendiculaire, mucocèle sinusale, mucocèle lacrymale), entraînant fréquemment un aspect kystique, pseudotumoral ».

${ }^{47}$ Cass. Civ. 1ère, 5 mai 1981, Gaz. Pal. 1981, 2, somm. 352.

${ }^{48}$ CSP Art. L1111-2, L1111-4.

${ }^{49}$ CSP Art. R4321-83, R4321-84.

${ }^{50}$ Cette phrase est parfois attribuée à Gandhi, parfois à Mandela.

${ }^{51}$ Dite Convention d'Oviedo, 4 avril 1997, https://rm.coe.int/168007cf99 ; L. n 2011-814 du 7 juill. 2011 relative à la bioéthique, art. $1^{\mathrm{er}}$ : «Est autorisée la ratification de la convention du Conseil de l'Europe pour la protection des droits de l'homme et de la dignité de l'être humain à l'égard des applications de la biologie et de la médecine : convention sur les droits de l'homme et la biomédecine, signée à Oviedo le 4 avril 1997 » [16-20].
} 
la nature de l'intervention ainsi que quant à ses conséquences et ses risques. / La personne concernée peut, à tout moment, librement retirer son consentement $»^{52}$.

Les textes de droit interne, ceux notamment relatifs aux droits des patients, sont animés du même esprit. L’information préalable et le consentement y sont particulièrement détaillés.

S'agissant de l'information, les dispositions de l'article L1111-2 du Code de la santé publique posent des jalons précis. L'information est à la fois un contenant et un contenu. Sur le premier point, l'information est «délivrée dans le cadre d'un entretien individuel ». L'information est donc orale, ce que confirment, à toutes fins utiles, les recommandations de la HAS ${ }^{53}$ [21]. Ces recommandations précisent que «La délivrance de l'information requiert du tact, du temps et de la disponibilité, ainsi qu'un environnement adapté. Elle s'inscrit dans un climat relationnel alliant écoute et prise en compte des attentes de la personne » [21].

S'agissant du « contenu », là encore le texte est précis : « Cette information porte sur les différentes investigations, traitements ou actions de prévention qui sont proposés, leur utilité, leur urgence éventuelle, leurs conséquences, les risques fréquents ou graves normalement prévisibles qu'ils comportent ainsi que sur les autres solutions possibles et sur les conséquences prévisibles en cas de refus $»^{54}$.

Il convient de rappeler que le risque de réalisation exceptionnelle doit être porté à la connaissance du patient : «la circonstance qu'un risque de décès ou d'invalidité répertorié dans la littérature médicale ne se réalise qu'exceptionnellement ne dispense pas les médecins de le porter à la connaissance du patient ${ }^{55}$.

Les débiteurs de l'obligation sont nombreux puisque celle-ci «incombe à tout professionnel de santé dans le cadre de ses compétences et dans le respect des règles professionnelles qui lui sont applicables $»^{56}$. Les recommandations de la HAS précisent que «Lorsque plusieurs professionnels de santé interviennent, chacun informe la personne des éléments relevant de son domaine de compétences en les situant dans la démarche générale de soin. Chaque professionnel de santé n'a pas à présumer que l'information relevant de ses compétences a été donnée par d'autres. Toutefois, il s'enquiert des informations déjà délivrées et en tient compte pour celles qu'il donne » [21].

\footnotetext{
52 https://rm.coe.int/168007cf99 ; Article 6 - Protection des personnes n'ayant pas la capacité de consentir ; Article 7 Protection des personnes souffrant d'un trouble mental; Article 8 - Situations d'urgence ; Article 9 - Souhaits précédemment exprimés.

${ }^{53} \mathrm{HAS}, \mathrm{p} 7$ [21] : «La délivrance de l'information, qui implique un dialogue, se fait toujours dans le cadre d'un entretien individuel». CSP Art. L1111-2, al.6: «Des recommandations de bonnes pratiques sur la délivrance de l'information sont établies par la Haute Autorité de santé et homologuées par arrêté du ministre chargé de la santé ».

${ }^{54}$ CSP Art. L1111-2, al.1.

${ }^{55}$ CE, 19 octobre 2016, n³91538 ; Vialla F. Revue Droit et Santé, n 75, 2017, 37-39 ; RGDM, n 62, 2017, 295-313, Responsabilité médicale, ss. Dir. J. Saison-Demars.

${ }^{56}$ CSP Art. L1111-2, al.2.
} 
Ajoutons que la preuve de l'information délivrée, contenant et contenu, incombe au professionnel ${ }^{57}$. Le Code de déontologie des masseurs-kinésithérapeutes ajoute quelques précisions utiles sur les devoirs du praticien en matière d'information : «Le masseur-kinésithérapeute, dans les limites de ses compétences, doit à la personne qu'il examine, qu'il soigne ou qu'il conseille, une information loyale, claire et appropriée sur son état, et les soins qu'il lui propose. Tout au long de la maladie, il tient compte de la personnalité du patient dans ses explications et veille à leur compréhension. Toutefois, sous réserve des dispositions de l'article L.1111-7, lorsque le médecin, appréciant en conscience, tient, pour des raisons légitimes, le patient dans l'ignorance d'un diagnostic ou pronostic graves, le masseur-kinésithérapeute ne doit pas révéler ces derniers $»^{58}$.

Il convient de marquer ici l'étonnement du juriste à la lecture de la dernière phrase de cet article qui semble induire que le masseur-kinésithérapeute pourrait être contraint de retenir une information, là où le texte légal ${ }^{59}$ n'envisage aucunement cette possibilité. Plus étonnant encore, le Code de déontologie des masseurs-kinésithérapeute ne semble pas avoir pris la mesure de la modification en 2012 du Code de déontologie des médecins ${ }^{60}$ qui rend l'article R4321-83 totalement obsolète sur ce point!

Au-delà de cette incongruité textuelle, il convient de retenir que l'on attend du masseurkinésithérapeute droiture, honnêteté et vérité ${ }^{61}$, ses propos doivent être aisés à comprendre ${ }^{62}$ et adaptés $^{63}$ à la personne destinataire.

\footnotetext{
${ }^{57}$ CSP Art. L1111-2, al.7. HAS, p8 [21]: «Le dossier contenant les informations de santé relatives à la personne mentionne les informations majeures qui lui ont été délivrées, par qui et à quelle date, ainsi que les difficultés éventuellement rencontrées lors de leur délivrance. Il mentionne le cas échéant les démarches entreprises lorsque la personne ne maîtrise pas suffisamment la langue française ou présente des difficultés de communication ou de compréhension. Ces mentions permettent aux autres professionnels de santé d'en prendre connaissance dans le but de favoriser la cohérence de l'information. Parce que ces mentions suffisent à servir de moyen de preuve en cas de litige, il n’y a pas lieu de demander à la personne une confirmation signée de la délivrance de l'information ».

${ }^{58}$ CSP Art. R4321-83.

${ }^{59}$ CSP Art.L1111-2.

${ }^{60}$ Depuis le Décret n ${ }^{\circ 2012-694}$ du 7 mai 2012 (art. 2), le Code de déontologie des médecins a été modifié et n'autorise plus cette réticence d'information envers le patient. L'alinéa 2 de l'article R4127-35 disposait antérieurement: «Toutefois, sous réserve des dispositions de l'article L. 1111-7, dans l'intérêt du malade et pour des raisons légitimes que le praticien apprécie en conscience, un malade peut être tenu dans l'ignorance d'un diagnostic ou d'un pronostic graves, sauf dans les cas où l'affection dont il est atteint expose les tiers à un risque de contamination ». Cet alinéa est désormais ainsi rédigé : "Toutefois, lorsqu'une personne demande à être tenue dans l'ignorance d'un diagnostic ou d'un pronostic, sa volonté doit être respectée, sauf si des tiers sont exposés à un risque de contamination »; Vialla $\mathrm{F}$. La réforme discrète du Code de déontologie médicale, A propos du Décret du 7 mai 2012, JCP 2012, éd. G, 663, p.1097 et s; Debost C, Giraudeau N, Véron P, Vialla F. RGDM 2012, n44, 231. On se doit toutefois de remarquer que le Code de déontologie des sages-femmes ménage toujours une telle possibilité (CSP Art. R4127-331, al.1) : «Sous réserve des dispositions de l'article L. 1111-7 et pour des raisons légitimes que la sage-femme apprécie en conscience, une patiente peut être laissée dans l'ignorance d'un diagnostic ou d'un pronostic grave la concernant. »; le même constat peut être dressé pour les chirurgiens-dentistes (CSP Art. R4127-239) : «Sous réserve des dispositions de l'article L. 1111-7 et pour des raisons légitimes que le chirurgien-dentiste apprécie en conscience, un patient peut être laissé dans l'ignorance d'un diagnostic ou d'un pronostic grave ».

${ }^{61}$ Dictionnaire de l'Académie Française, $V^{\circ}$ LOYAUTÉ : «n. $f . X I^{e}$ siècle. Dérivé de loyal. Respect de la vérité, fidélité à la parole donnée, aux engagements pris; droiture, honnêteté », https://academie.atilf.fr; Le devoir de loyauté explique que l'information dispensée doit demeurer circonscrit au champ de compétences de chaque professionnel : CSP Art. L1111-2, Art. R4321-83.
} 
Dès lors que l'information délivrée répond à ces exigences, le patient voit son jugement éclairé et il est à même d'exprimer son consentement.

Le terme consentement, quant à lui, signifie tout à la fois l' «Action d'accepter, de donner son accord ${ }^{64}$ et le «résultat de cette action ${ }^{64}$, il caractérise 1' «adhésion d'une partie à la proposition de l'autre ${ }^{64}$.

La notion semble, en apparence, assez aisée à saisir. Positivement entendu, le consentement caractérise la manifestation de volonté qui entérine l'adhésion du patient aux préconisations du soignants ${ }^{65}$. Négativement appréhendé il exprime le refus de patient ${ }^{66}$.

Mais la notion de consentement se révèle plus complexe qu'il n'y parait de prime abord. Il est susceptible de plusieurs approches. Selon St. Thomas d'Aquin : «consensus autem voluntatis est actus qui praesupponit actum intellectus » [22], cette proposition se retrouve dans l'adage juridique «L'acte de volonté présuppose un acte intellectuel» [23]. Consentir n'est pas un acte passif mais celui d'un agent dont il convient de « hausser le niveau de compréhension » afin qu'il soit capable de produire «l'acte intellectuel » envisagé. Cette première appréhension du consentement conduit à priver de valeur juridique la volonté de tous ceux dont le discernement est altéré : «le consentement est juridiquement inexistant si l'intelligence fait défaut. Ainsi en est-il de l'enfant en bas âge privé de raison, [...] du vieillard dont les facultés intellectuelles sont si affaiblies qu'il ne saurait avoir exprimé une volonté raisonnable... »[23]. Le consentement ainsi défini est celui du droit des obligations et des contrats. Or il convient de s'interroger sur la pertinence de l'application de cette approche particulière du consentement à l'acte de soin. On sait que le lien unissant le professionnel de santé, libéral, et le patient se voit, depuis l'arrêt Dame Mercier ${ }^{67}$, qualifiée de «véritable contrat ». Mais l'approche contractuelle de la relation de soin est aujourd'hui remise en cause par

${ }^{62}$ Dictionnaire de l'Académie Française, $\mathrm{V}^{\circ}$ CLAIR, -AIRE adj. et n. XI ${ }^{\mathrm{e}}$ siècle. Du latin clarus, «clair, brillant, sonore, intelligible ».I. Adj.B. Au sens figuré. 1. Qu'il est aisé de comprendre; intelligible, limpide ; V ${ }^{\circ} \mathrm{CLARTÉ}^{\mathrm{C}}$ «II. Au sens figuré. 1. Caractère de ce qui est clair intellectuellement, de ce qu'il est aisé de comprendre»; https://academie.atilf.fr

${ }^{63}$ Dictionnaire de l'Académie Française, $\mathrm{V}^{\circ}$ APPROPRIER « 1. V. tr. Adapter à, rendre propre à. ».

64 Dictionnaire de l'Académie Française, V ${ }^{\circ}$ CONSENTEMENT n. m. XII ${ }^{\mathrm{e}}$ siècle. Dérivé de consentir, https://academie.atilf.fr

${ }^{65}$ CSP Art. L1111-4, al.1.

${ }^{66}$ CSP Art.L1111-4, al. 2 : «Toute personne a le droit de refuser ou de ne pas recevoir un traitement [...]», al.3: « [...] Si, par sa volonté de refuser ou d'interrompre tout traitement, la personne met sa vie en danger, elle doit réitérer sa décision dans un délai raisonnable [...]», al 4 : «Aucun acte médical ni aucun traitement ne peut être pratiqué sans le consentement libre et éclairé de la personne et ce consentement peut être retiré à tout moment»; CSP Art. R432184 : «[...] Lorsque le patient, en état d'exprimer sa volonté, refuse le traitement proposé, le masseur-kinésithérapeute respecte ce refus après avoir informé le patient de ses conséquences et, avec l'accord de ce dernier, le médecin prescripteur $\gg$.

${ }^{67}$ Civ. 20 mai 1936, DP 1936, 1, 88 : «Mais attendu qu'il se forme entre le médecin et son client un véritable contrat comportant pour le praticien, l'engagement, sinon, bien évidemment, de guérir le malade, ce qui n'a d'ailleurs jamais été allégué, du moins de lui donner des soins, non pas quelconques, ainsi que paraît l'énoncer le moyen du pourvoi, mais consciencieux, attentifs, et réserve faite des circonstances exceptionnelles, conformes aux données acquises de la science ; que la violation, même involontaire, de cette obligation contractuelle, est sanctionnée par une responsabilité 
une partie de la doctrine [9, 24-26]. Assurément, «Il existe bien un accord entre le patient et son médecin. Mais cet accord est-il vraiment créateur d'obligations à la charge du médecin ? [...] Lorsqu'un particulier a recours au service d'un médecin, [...] outre le fait qu'il lui demande un service et s'engage à le rémunérer, il ne discute pas avec lui des obligations auxquelles il entend que le professionnel soit soumis. [...] Il est donc artificiel de rattacher des prétendues obligations d'information ou de sécurité qui seraient de l'essence d'un contrat médical, dans la mesure où c'est la loi qui prescrit ces normes de comportement et non pas l'accord de volonté des parties. [...] Puisque la liberté et la volonté individuelle n'ont pas participé à la création des obligations du professionnel, puisque ces obligations ne sont pas d'origine contractuelle, il est difficile de voir dans leur violation une responsabilité contractuelle » [27].

Le consentement à l'acte de soin n'est pas soluble dans la définition thomiste précédemment proposée, il présente une nature complexe, non pas figée dans une approche « obligationnelle » mais ancrée dans les droits fondamentaux de la personne ${ }^{68}$.

On peut alors considérer que «le consentement dont il s'agit n'est pas celui du droit civil des obligations, c'est une garantie procédurale qui oblige les médecins à respecter un droit fondamental de la personne, celui que traduit le vieil adage noli me tangere $»[28]$. «Noli me tangere ${ }^{69}$ est la

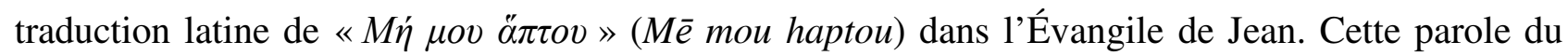
Christ ressuscité à Marie-Madeleine est, aujourd'hui encore, considérée comme le fondement des principes juridiques d'inviolabilité et d'indisponibilité du corps humain ${ }^{70}$.

Le consentement, et l'information qui le précède, ne relèvent donc pas d'une approche contractualiste mais d'un souci de garantir à chacun le respect de ses droits fondamentaux et particulièrement le droit au respect de son corps. Aucune atteinte à l'intégrité corporelle ne peut se justifier si elle n'est consentie.

de même nature; -également contractuelle»; V. Les grandes décisions du droit médical, LGDJ $20142^{\mathrm{e}}$ Ed. Dir. Vialla $\mathrm{F}$.

${ }^{68}$ Dignité, indisponibilité et inviolabilité du corps humain.

${ }^{69}$ Ioannes 20 :17 Biblia Sacra Vulgata (VULGATE) «Dicit ei Jesus: Noli me tangere, nondum enim ascendi ad Patrem meum [...] »; Jean 20,17 «Jésus lui dit : Ne me touche pas; car je ne suis pas encore monté vers mon Père. ».

${ }^{70}$ Code civil Art. Art. 16-1 : «Chacun a droit au respect de son corps. / Le corps humain est inviolable. / Le corps humain, ses éléments et ses produits ne peuvent faire l'objet d'un droit patrimonial ». Art. 16-1-1 : «Le respect dî au corps humain ne cesse pas avec la mort. /Les restes des personnes décédées, y compris les cendres de celles dont le corps a donné lieu à crémation, doivent être traités avec respect, dignité et décence»; Art. 16-2: «Le juge peut prescrire toutes mesures propres à empêcher ou faire cesser une atteinte illicite au corps humain ou des agissements illicites portant sur des éléments ou des produits de celui-ci, y compris après la mort. »; Art. 16-3 : «Il ne peut être porté atteinte à l'intégrité du corps humain qu'en cas de nécessité médicale pour la personne ou à titre exceptionnel dans l'intérêt thérapeutique d'autrui./ Le consentement de l'intéressé doit être recueilli préalablement hors le cas où son état rend nécessaire une intervention thérapeutique à laquelle il n'est pas à même de consentir. ». 
Cet ancrage dans les droits et libertés fondamentaux explique qu'aujourd'hui la loi consacre la face négative du consentement, autrement dit le refus de $\operatorname{soin}^{71}$, qui peut aller jusqu'à l'engagement de son pronostic vital par le patient ${ }^{72}$.

Que le consentement soit envisagé sous l'angle du droit des obligations ou celui des droits fondamentaux, l'information préalable demeure exigible. Cette information est destinée à permettre l'adhésion du patient à l'atteinte envisagée à son intégrité.

L'information doit permettre une «métamorphose » du patient qui ignorant des choses de l'Art doit voir son niveau de compréhension haussé afin que sa parole ne soit pas circonscrite à un « oui » mais devienne un « oui parce que ».

Cette approche permet de mieux comprendre pourquoi l'information exigée confine à l'exhaustivité ${ }^{73}$, pourquoi elle doit être progressive ${ }^{74}$, loyale, claire et appropriée.

Cela permet aussi d'expliquer que l'information n'est pas une obligation isolée, elle est complétée par le devoir de conseil ${ }^{75}$, par le souci de voir le praticien expliquer ${ }^{76}$, veiller à la compréhension ${ }^{77}$ des informations dispensées et, surtout par l'obligation d'émettre propositions ${ }^{78}$ et préconisation ${ }^{79}$.

Cette analyse renouvelée de la nature du consentement est essentielle et elle n'est pas sans conséquence lorsque l'on aborde les situations pour lesquelles peuvent être envisagées une exception à l'information et au recueil du consentement.

La «fondamentalisation» de l'information et du consentement conduit à rendre incontournable le respect de la parole du patient, peut-on alors envisager qu'en certaines occurrences, le professionnel de santé puisse passer outre et pratiquer un acte sans avoir à informer la personne et en se dispensant de s'enquérir de sa volonté ? Lorsque l'approche de la notion de consentement, et de son préalable l'information, demeure celle du droit des obligations de telles exceptions peuvent aisément être envisagées et justifiées. Tout ce qui altère la volonté, l'âge, le trouble mental etc., justifie que, dans l'intérêt de la personne, le soignant puisse passer outre et effectuer les actes qu'il

\footnotetext{
${ }^{71}$ CSP Art. L1111-4 ; Art. R4321-84.

${ }^{72}$ CSP Art. L1110-5-2-2.

${ }^{73}$ CSP Art. L1111-2 : «Cette information porte sur les différentes investigations, traitements ou actions de prévention qui sont proposés, leur utilité, leur urgence éventuelle, leurs conséquences, les risques fréquents ou graves normalement prévisibles qu'ils comportent ainsi que sur les autres solutions possibles et sur les conséquences prévisibles en cas de refus ». HAS : «Celui-ci permet d'ajouter aux informations génériques des éléments adaptes à la situation de la personne, ainsi que de répondre aux questions qu'elle se pose, et de lui permettre d'exprimer ses préférences $\gg[21]$.

${ }^{74} \mathrm{HAS}$ : «La délivrance de l'information requiert du tact, du temps et de la disponibilité, ainsi qu'un environnement adapté. Elle s'inscrit dans un climat relationnel alliant écoute et prise en compte des attentes de la personne. Il est souvent nécessaire de délivrer l'information de façon progressive et en plusieurs fois. Elle est réitérée à chaque fois que cela est nécessaire et elle est régulièrement actualisée » [21].
}

${ }^{75}$ CSP Art. R4321-83.

${ }^{76}$ CSP Art. R4321-83.

${ }^{77}$ CSP Art. R4321-83.

${ }^{78}$ CSP Art. R4321-83.

${ }^{79}$ CSP Art. L1111-4, al. ${ }^{\mathrm{er}}$. 
juge nécessaires. Lorsqu'à la nature contractuelle s'ajoute voire se substitue une analyse assise sur les droits fondamentaux de la personne, la dispense est plus délicate à justifier puisqu'elle conduit à remettre en cause la qualité même de personne, de sujet de droit !

\section{II/ l'urgence créatrice d'une exception aux devoirs d'information et de recueil du consentement ?}

Le poète Scarron considérait qu' «en chose où le péril paraît de tous côtés, on peut fort bien passer sur les formalités ${ }^{80}$. L'urgence lorsqu'elle est toujours synonyme de péril pour la personne, peutelle justifier que des actes soient pratiqués sans une information préalable et, donc, sans souci de recueillir et de respecter la volonté de la personne?

Le textes permettent d'entrevoir qu'en fonction des circonstances des exceptions peuvent bien exister.

L'alinéa deux de l'article 16-3 du Code civil dispose que «Le consentement de l'intéressé doit être recueilli préalablement hors le cas où son état rend nécessaire une intervention thérapeutique à laquelle il n'est pas à même de consentir ». Ce texte légitime l'action en absence de consentement lorsqu'un acte thérapeutique est nécessaire (non pas simplement utile) et que la personne n'est pas à même de consentir. Il n'est pas ici expressément question d'urgence. L'exception est moins liée à la situation d'urgence qu'à une circonstance propre au patient qui n'est pas en situation d'exprimer sa volonté ou d'émettre un consentement. Assurément les deux situations peuvent se recouper, on peut parfaitement envisager qu'en cas d'urgence le patient puisse ne pas être à même de consentir.

C'est surtout dans les dispositions de l'article L1111-2, alinéa 2, que l'on découvre une réelle exception à l'obligation de délivrer l'information : «Cette information incombe à tout professionnel de santé dans le cadre de ses compétences et dans le respect des règles professionnelles qui lui sont applicables. Seules l'urgence ou l'impossibilité d'informer peuvent l'en dispenser ».

Quelques remarques doivent être émises sur les exceptions légales expressément affirmées.

Ce sont deux hypothèses, et deux seulement ${ }^{81}$, qui sont émises. La hiérarchie des normes [29-31] conduit à remettre en question toutes autres exceptions non prévues par la loi et, notamment, celle déjà envisagée d'une non révélation de diagnostic ou de pronostic grave ${ }^{82}$. Sont donc visées

\footnotetext{
${ }^{80}$ Scarron, Don Japhet d'Arménie, Acte 5 sc. 1.

${ }^{81}$ Auxquelles s'ajoute celle figurant à l'alinéa 4 de l'article L1111-2 : «La volonté d'une personne d'être tenue dans l'ignorance d'un diagnostic ou d'un pronostic doit être respectée, sauf lorsque des tiers sont exposés à un risque de transmission ». On notera avec étonnement que le Code de déontologie médicale prévoit cette exception avec, cependant, une modification, contestable : CSP Art R4127-35, al. 2, «Toutefois, lorsqu'une personne demande à être tenue dans l'ignorance d'un diagnostic ou d'un pronostic, sa volonté doit être respectée, sauf si des tiers sont exposés à un risque de contamination ». Substituer « contamination » à «transmission » n'est pas sans conséquence !

${ }^{82}$ Voir supra. Spéc. note 60.
} 
«l'urgence ou l'impossibilité d'informer». La seconde semble s'apparenter à la situation prévue par l'article 16-3, alinéa 2, du Code civil ${ }^{83}$.

L'impossibilité d'informer demeure une notion difficile à cerner. Le dictionnaire de l'Académie Française définit ainsi le terme «impossibilité » : «Le fait qu'une action soit impossible, [...] Se trouver dans l'impossibilité matérielle de faire quelque chose. [...] Spécialt. Impossibilité physique, le fait qu'une chose soit impossible selon l'ordre de la nature ${ }^{84}$.

On peut considérer que "L'état d'inconscience est ici visé. L'impossibilité matérielle de recevoir l'information médicale, préalable juridique au consentement, fonde cette limite » [32]. Il convient ici de relever que les dispositions de l'article L1111-4 du Code de la santé publique relayent implicitement pour le consentement ${ }^{85}$, les deux exceptions de l'article L1111-2 relatif à l'information. L'urgence et l'impossibilité permettent de se dispenser d'informer et de recueillir le consentement de la personne, sous réserve de consulter des tiers. L'alinéa 4 de l'article L1111-4 précise que «Lorsque la personne est hors d'état d'exprimer sa volonté, aucune intervention ou investigation ne peut être réalisée, sauf urgence ou impossibilité, sans que la personne de confiance prévue à l'article L.1111-6, ou la famille, ou à défaut, un de ses proches ait été consulté ${ }^{86}$.

Dans le même esprit l'article 8 de la Convention Européenne pour la protection des Droits de l'Homme et la dignité de l'être humain à l'égard des applications de la biologie et de la médecine (Convention sur les droit de l'homme et la biomédecine) ${ }^{51}$ prévoit que «Lorsqu'en raison d'une situation d'urgence le consentement approprié ne peut être obtenu, il pourra être procédé immédiatement à toute intervention médicalement indispensable pour le bénéfice de la santé de la personne concernée ».

Les termes utilisés permettent de souligner les contours de l'exception. L'intervention doit, tout d'abord, être médicalement indispensable et non simplement utile pour que puisse être réalisé un acte immédiat. Dès lors, en sont «exclues les interventions pour lesquelles un délai est acceptable » [33]. Lorsque l'acte peut être différé, en dépit de la situation d'urgence, le praticien doit s'abstenir. Cette analyse doit aussi être menée pour la mise en œuvre des dispositions de l'article L4321-1 du Code de la santé publique. Le masseur-kinésithérapeute n'est habilité à agir, en l'absence de médecin, que pour accomplir «les premiers actes de soins nécessaires en masso-kinésithérapie ». Hors nécessité il doit différer son action. Son appréciation de l'urgence et de l'indispensabilité de

\footnotetext{
83 «...] hors le cas où son état rend nécessaire une intervention thérapeutique à laquelle il n'est pas à même de consentir».

${ }^{84}$ Dictionnaire de l'Académie Française, $V^{\circ}$ IMPOSSIBILITÉ n. f. xiiie siècle, impossibleté. Emprunté du latin impossibilitas, de même sens. https://academie.atilf.fr

${ }^{85}$ Depuis la rédaction de la loi éponyme du 4 mars 2002, Loi n ${ }^{\circ 2002-303 ~ d u ~} 4$ mars 2002, JORF 5 mars 2002, dite loi Kouchner.
} 
l'acte étant de nature à engager sa responsabilité. Ce temps accordé a une double vertu : il évite toute précipitation mais permet aussi d'envisager la possibilité ultérieure de voir le patient être en capacité de recevoir l'information et d'émettre son consentement.

On relèvera, ensuite, l'emploi du verbe « pouvoir » (« il pourra être procédé ») préféré à « devoir ». Une marge d'appréciation est laissée au professionnel de santé. La même analyse peut être menée au regard de l'article L1111-2: «Seules l'urgence ou l'impossibilité d'informer peuvent l'en dispenser ».

Il convient cependant de s'interroger sur le fait de savoir si «l'impossibilité » visée aux articles L1111-2 (information) et L1111-4 (consentement) se limite à une impossibilité matérielle. Le Dictionnaire de l'Académie Française, en effet, envisage l'impossibilité morale qu'il définit comme «le fait qu'une chose paraisse impossible, étant donné les conjectures fondées sur la nature humaine ${ }^{87}$. Peut-on ainsi envisager une impossibilité d'informer et de recueillir le consentement d'une nature plus subjective, telle que celle liée à la fragilité psychologique présentée par un patient. La négative semble devoir s'imposer. L'évolution de l'article R4127-35 du Code de la santé publique semble confirmer cette position. Nous avons que ce texte autorisait, jusqu'en 2012, le médecin à ne pas révéler un diagnostic ou un pronostic grave. Ce «privilège thérapeutique », selon la formule québécoise, a désormais disparu ${ }^{88}$. Il fait place à la possibilité pour le patient de refuser de recevoir l'information, notamment celle relative à un diagnostic ou un pronostic, sous réserve que des tiers ne soient pas exposés à un risque de transmission ${ }^{89}$.

Ainsi, l'urgence ou l'impossibilité d'informer peuvent «dispenser»le professionnel de santé d'avoir à délivrer l'information et à recueillir son consentement. Il faut noter que l'article L1111-2 utilise le verbe «dispenser». Le terme peut être ainsi défini : «II. Exempter d'une obligation. 1. Faire une exception à la règle ou à la loi en accordant une dispense à quelqu'un. [...] 2. Dégager quelqu'un d'une obligation, d'une activité considérée comme pénible $»^{90}$. La dispense est donc une «Exemption, acte par lequel une autorité permet à un demandeur de se soustraire à une règle établie, soit qu'il fasse ce qui est normalement prohibé, soit qu'il ne fasse pas ce qui est prescrit $\gg^{91}$.

\footnotetext{
${ }^{86}$ CSP Art. R4321-84, al.2 : «Si le patient est hors d'état d'exprimer sa volonté, le masseur-kinésithérapeute ne peut intervenir sans que la personne de confiance désignée ou ses proches aient été prévenus et informés, sauf urgence ou impossibilité $[\ldots] »$.

87 Dictionnaire de l'Académie Française, $V^{\circ}$ IMPOSSIBILITÉ n. f. xiiie siècle, impossibleté. Emprunté du latin impossibilitas, de même sens. https://academie.atilf.fr

${ }^{88}$ Sur le maintien de cette possibilité dans les Codes de déontologie des sages-femmes, des chirurgiens-dentistes et, indirectement, des masseurs-kinésithérapeutes [voir supra, notamment note 60].

${ }^{89}$ Le texte légal (L1111-2) mentionne le risque de transmission, le Code de déontologie médicale (R4127-35) se réfère au risque de contamination.

${ }^{90}$ Dictionnaire de l'Académie Française, $V^{\circ}$ DISPENSER. https://academie.atilf.fr

${ }^{91}$ Dictionnaire de l'Académie Française, $V^{\circ}$ DISPENSER DISPENSE n. f. XVI ${ }^{\mathrm{e}}$ siècle. Déverbal de dispenser ; https://academie.atilf.fr
} 
C'est bien une exception au principe qui est affirmée par les textes. Il faut alors rappeler l'importance de l'adage «Exceptio est strictissimae interpretationis». Les exceptions sont d'interprétation stricte! Assurément «une exception [...] mérite d'être appliquée dans toute la mesure de sa raison d'être » [34] mais si l'interprétation ne doit pas être restrictive, elle doit demeurer stricte.

Il convient alors de bien identifier le contexte précis pour lequel peut être envisagée le jeu de l'exception qui nous préoccupe: la dispense d'information et conséquemment de recueil du consentement. Plus précisément il s'agira de s'interroger sur la possibilité de voir l'exception mise en œuvre par le masseur-kinésithérapeute dans le cadre des dispositions de l'article L4321-1 du Code de la santé publique: «En cas d'urgence et en l'absence d'un médecin, le masseurkinésithérapeute est habilité à accomplir les premiers actes de soins nécessaires en massokinésithérapie. Un compte rendu des actes accomplis dans ces conditions est remis au médecin dès son intervention $»^{92}$.

Confronté à une situation d'urgence, le masseur-kinésithérapeute est habilité par la loi à accomplir, sous réserve de l'absence d'un médecin, un nombre limité d'actes (les premiers actes de soins en masso-kinésithérapie). On notera que pèse sur lui le devoir d'informer non pre ou per mais post... le médecin. Lequel ? Le texte est pour le moins imprécis, mais la traçabilité des actes est un impératif légal!

En revanche, le texte est muet sur les questions de l'information due et sur le consentement de la personne soignée. Est-ce à dire que l'article L4321-1 du Code de la santé publique se fond, sur ce point, dans les dispositions des articles L1111-2 et L1111-4 ?

Le masseur-kinésithérapeute peut-il pratiquer ces actes nécessaires sans préalablement informer le patient et sans recueillir son adhésion à la réalisation des actes envisagés.

La dispense légale des articles L1111-2 et L1111-4 joue-t-elle?

\section{Vexata questio}

Quel est alors ce contexte d'urgence en masso-kinésithérapie dont il est ici question.

Dans l'étymologie latine, urgens $^{93}$ signifie pressant. La notion d'urgence est complexe et désigne «Dans le domaine médical, [la] situation d'un patient à soigner sans délais ${ }^{94}$.

\footnotetext{
92 CSP Article L4321-1 Modifié par Loi n²016-41 du 26 janvier 2016 - art. 123 Modifié par Loi n²016-41 du 26 janvier 2016 - art. 134.

${ }_{93}$ Participe présent adjectivé d'urgeo : presser, insister avec opiniâtreté.

${ }^{94}$ Dictionnaire médical de l'Académie Nationale de Médecine, $\mathrm{V}^{\circ}$ urgence; http://dictionnaire.academiemedecine.fr/index.php?q=urgence
} 
De quelle urgence s'agit-il ? L'urgence vraie, analysée sur la base du savoir du soignant, ou celle qualifiée de réelle, plus subjective, ressentie par le patient ?

Le dictionnaire médical de l'Académie Nationale de Médecine souligne, en effet, que «La notion médicale fait intervenir deux acteurs antagonistes, celui qui appelle à l'aide et celui qui est sollicité : chacun apprécie différemment la situation. L'appellant (sic) ressent l'urgence de façon surtout subjective, c'est l'urgence ressentie. Celui qui est sollicité apprécie plus objectivement et cherche à distinguer l'urgence vraie de la fausse urgence $»^{94}$.

Cette notion de « ressenti » est aussi présente dans la Circulaire DH.DGS-90.326 du 15 février 1990 relative à l'amélioration de l'accueil des urgences qui introduit la notion «d'urgence ressentie » dans les missions d'accueil du service public hospitalier. Ce texte souligne que «les usagers ont aujourd'hui tendance à confondre soins non programmés ou soins ressentis comme urgents et soins urgents ». Le texte aborde aussi la question des «Consultations non programmées en établissement de santé »[35]:

- «Dans certaines régions, le besoin de service public saisonnier et le besoin de proximité lié au délai d'accès aux soins dans ces zones du territoire, ont incité au développement de consultations non programmées au sein des établissements de santé, autorisés ou non à l'accueil des urgences, qui permettent d'accueillir des patients demandeurs de soins non programmés ressentis comme urgents. Ces consultations ne sont pas assurées par des médecins urgentistes.

- Ces consultations accueillent les patients venus soit directement, soit adressés par le centre 15 ou par les médecins libéraux du secteur ».

Assurément, « Si les urgences soignent des cas ordinaires, c'est qu'une longue histoire a fait naître dans les populations urbaines la perception d'une offre de soins rapides» [36,37], mais précisément, faut-il s'inscrire dans cette logique et étendre la notion aux demandes de soins non programmés ressentis comme urgents par les patients ? Sur ce point, on peut relever que l'Ordre National des masseurs-kinésithérapeutes semble l'envisager favorablement dans sa «campagne de communication sur l'accès direct et l'urgence en kinésithérapie rendu possible par la loi de modernisation de notre système de santé du 26 janvier $2016 »{ }^{95}$. On peut lire sur l'affiche présente sur le site de l'Ordre : «6000 entorses de la cheville par jour. Urgences saturées > accès direct au $k i n e^{*} »$. Opportunisme ou provocation, la question est quelque peu détournée de sa finalité première, puisqu'elle est présentée sous l'angle de l'accès direct permettant de palier la saturation des services d'urgence des établissements [38].

Alertée par les dérives potentielles, l'Académie de médecine a adressé conjointement à Madame la Ministre de la Santé et au CNOMK, un courrier dont la conclusion lapidaire est on ne peut plus

${ }^{95}$ http://www.ordremk.fr/actualites/ordre/acces-direct-en-kinesitherapie-le-cas-de-lurgence/ 
claire: «Il n’y a pas d'urgence en kinésithérapie ni en médecine, il n’y a que des patients pressés $\gg$.

L'urgence doit-elle être abordée sous l'angle de ses conséquences? Elle conduit, alors, à appréhender le risque qui existe à différer une action et, juridiquement elle confine à l'omission de porter secours. Les soignants ne sont pas les seuls à s'interroger sur la notion d'urgence [33,39-41]. Ainsi, le vocabulaire juridique [42] définit-il l'urgence comme le «caractère d'un état de fait susceptible d'entraîner, s'il n'y est porté remède à bref délai, un préjudice irréparable ». Certains juristes relèvent la difficulté, voire l'impossibilité à apporter une définition satisfaisante : «définir l'urgence d'une façon rigoureuse est une entreprise vouée à l'échec. La plupart des auteurs qui s'y sont essayé n'ont abouti qu'à des formules imprécises qui gravitent autour de l'idée d'un préjudice dans le retard $\gg[43]$.

Relevons simplement que la question de la temporalité semble constituer la pierre angulaire de la question, il convient alors de rappeler que les acteurs de santé doivent pouvoir et savoir distinguer

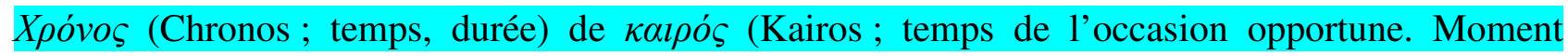

\section{opportun).}

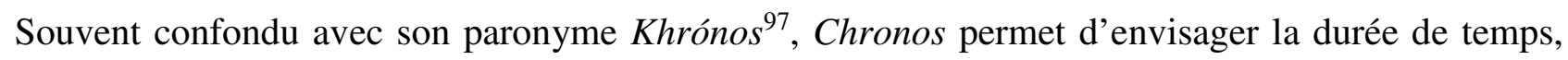
passé, présent et futur. Avec кaı ó $\varsigma^{98}$, il ne s'agit plus d'une dimension temporelle linéaire, comme avec Chronos, mais de la mesure du moment opportun. Assurément, Kairos est une mesure du temps contextualisé, mais il est aussi porteur d'autres considérations, la «juste mesure », «l'efficacité ». Kairos induit aussi la juste mesure et la convenance, il doit concilier temps et efficacité de l'action humaine. On comprend que l'appréhension du Chronos et du Kairos soit essentielle lorsqu'il s'agit d'envisager l'encadrement et la régulation du soin d'urgence. Le temps

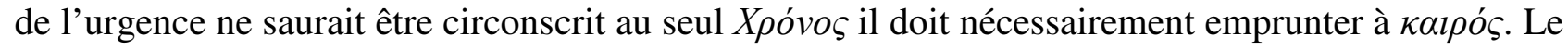
Dictionnaire Médical de l'Académie Nationale de Médecine souligne ainsi qu' «Après avoir obtenu les renseignements permettant un premier bilan fonctionnel et lésionnel, le médecin apprécie si l'urgence est vraie et, si oui, quel est le temps nécessaire pour être efficace » ${ }^{99}$.

Si la situation, évaluée et appréciée, par le professionnel de santé peut être qualifiée «d'urgence vraie » alors l'action est légitime voire obligatoire afin de sauvegarder l'intégrité de la personne nonobstant l'absence de recueil consentement : «Reconnu comme principe fondamental, le recueil du consentement ne jouit pas pour autant d'un caractère absolu [...] Une telle situation se rencontre en cas d'urgence médicale. La notion d'urgence opère une altération des règles traditionnelles de l'exercice médical [...] L'urgence médicale concerne une situation où le

\footnotetext{
96 cité par [38].

${ }^{97}$ Krónos ; Cronos roi des Titans et père de Zev́s — Zeús.

${ }^{98}$ Kairos.

${ }^{99}$ http://dictionnaire.academie-medecine.fr/index.php?q=urgence
} 
pronostic vital ou fonctionnel d'une personne est engagé » [41]. On conçoit que si «l'urgence en droit médical est une situation associant un pronostic vital engagé et une absence d'alternative thérapeutique » [41] cela conduise à considérer que «La protection de la vie apparaît alors comme une valeur supérieure à celle de la volonté » [41].

L'analyse de l'article 8 de la Convention d'Oviedo est, ici aussi, d'un précieux secours. Le texte «permet de déroger à la règle générale de recueil du consentement posée aux articles 5 et 6 , en soulignant le conflit de devoirs qui se pose alors [...] la dérogation au principe du consentement est limitée aux situations d'urgence qui empêchent le praticien d'obtenir le consentement approprié [...] l'exposé des motifs cite le cas d'un patient dans le coma [...] il n'est pas explicite que le médecin se trouve dispensé de la recherche du consentement face à une personne en état d'exprimer sa volonté pour la seule raison que le médecin n'aurait pas le temps de la lui demander. L'exposé des motifs, sans être ni tranché ni contraignant dans sa formulation, indique toutefois l'orientation qui prévaut aux yeux des auteurs du texte: "Les membres des professions de santé devraient cependant, même dans une situation d'urgence, mettre en auvre des mesures raisonnables pour déterminer quels pourraient être les souhaits du patient » [44]. » [33].

Une frontière apparaît perceptible quant à la possible dispense de l'information et de recueil du consentement en cas d'urgence. Sont regroupées sous la qualification « d'urgence » des situations de nature bien distinctes.

Dans certaines hypothèses l'urgence « constitue une situation où la vie du patient est en jeu, et dans laquelle il est hors d'état d'exprimer son consentement »[41], dans d'autres occurrences, moins dramatiques, l'état préoccupant du patient n'est pas cependant de nature à engager son pronostic vital.

La dispense prévue par les textes ne s'appliquerait que pour les premières circonstances : «Si l'urgence vitale est démontrée, et uniquement dans cette hypothèse, l'atteinte au droit de donner son consentement est tolérable. Néanmoins, aucune exception au principe ne permet de déroger de manière systématique au respect de la volonté du malade. Si cette nécessité vitale n'est pas caractérisée, le médecin se doit de respecter les choix du patient » [41].

Réserver le jeu de l'exception aux seules situations de péril vital semble davantage relever d'une analyse de bon sens que d'une lecture imposée par les textes. Ni l'article L1111-2 ni l'article L1111-4 ne se réfèrent à «l'urgence vitale». Le premier ${ }^{100}$ précise que «Seules l'urgence ou l'impossibilité d'informer peuvent l'en dispenser », le second ${ }^{101}$ dispose que «Lorsque la personne est hors d'état d'exprimer sa volonté, aucune intervention ou investigation ne peut être réalisée, sauf urgence ou impossibilité [...]».

\footnotetext{
${ }^{100}$ Relatif à l'obligation d'information.

${ }^{101}$ Relatif au consentement.
} 
La dispense d'information est rédigée en des termes plus larges que l'exception faite au recueil du consentement. Peut-on pour autant dissocier les deux questions en affirmant que l'urgence permettrait toujours l'absence d'information ${ }^{102}$, alors que le recueil du consentement s'imposerait, excepté «Lorsque la personne est hors d'état d'exprimer sa volonté ». Cette proposition nous semble devoir être exclue dans la mesure où, nous l'avons vu, l'information est un préalable au consentement et qu'un consentement non éclairé n'aurait aucune valeur. Si le consentement est requis, ce qui semble bien être commandé par l'article L1111-4 dès lors que la personne peut s'exprimer, alors l'information est due.

La circonstance d'urgence vitale appelle d'autres précisions. Une telle situation impose, au professionnel de santé notamment, un devoir incontournable d'action, pénalement « sanctionnable ${ }^{103}$. Le devoir d'assistance est d'ailleurs expressément prévu par la déontologie des masseurs-kinésithérapeutes : «Le masseur-kinésithérapeute qui se trouve en présence d'un malade ou d'un blessé en péril ou, informé qu'un malade ou un blessé est en péril, lui porte assistance ou s'assure qu'il reçoit les soins nécessaires » ${ }^{104}$.

On peut donc considérer que les dispositions de l'article L4321-1 du Code de la santé publique visent principalement des situations d'urgence non vitale ${ }^{105}$. Aussi la plus extrême prudence doit être recommandée au masseur-kinésithérapeute qui envisagerait de mettre en œuvre l'habilitation ouverte par l'article L4321-1. Dès lors que le patient est en mesure d'exprimer sa volonté se dispenser de l'informer et de recueillir son consentement paraît exclu. Si le patient est hors d'état de s'exprimer il n'est pas certains que les actes de masso-kinésithérapie soient la priorité du professionnel de santé lui portant assistance.

Dans la majorité des cas, le masseur-kinésithérapeute confronté à la situation d'urgence envisagée par l'article L4321-1 aura face à lui un patient qui, s'il peut avoir besoin, voire s'il sollicite une intervention, n'est pas pour autant confronté à un risque majeur pour sa santé et sa vie.

On envisage difficilement que ce patient, conscient, soit soigné sans information préalable adaptée à la situation, ni que le praticien puisse mettre un œuvre un acte que le patient refuserait. On conçoit cependant que l'information dispensée dans ce type de circonstances puisse être relativement moins développée. C'est au demeurant le sens des termes de l'article R4321-83 du Code de la santé

\footnotetext{
${ }^{102}$ En appliquant la maxime «ubi lex non distinguit nec nos non distinguere debemus » : « Où la loi ne distingue pas, nous ne devons pas distinguer $\gg$.

103 Code pénal Art. 226-3 al. 2 et 3 : «Sera puni des mêmes peines [cinq ans d'emprisonnement et de 75000 euros d'amende] quiconque s'abstient volontairement de porter à une personne en péril l'assistance que, sans risque pour lui ou pour les tiers, il pouvait lui prêter soit par son action personnelle, soit en provoquant un secours. / Les peines sont portées à sept ans d'emprisonnement et 100000 euros d'amende lorsque le crime ou le délit contre l'intégrité corporelle de la personne mentionnée au premier alinéa est commis sur un mineur de quinze ans ou lorsque la personne en péril mentionnée au deuxième alinéa est un mineur de quinze ans ». Article R4321-60: «Le masseurkinésithérapeute qui se trouve en présence d'un malade ou d'un blessé en péril ou, informé qu'un malade ou un blessé est en péril, lui porte assistance ou s'assure qu'il reçoit les soins nécessaires ».

${ }^{104}$ CSP Art. R4321-60.
} 
publique lorsqu'il envisage une information «appropriée sur son état, et les soins qu'il lui propose».

Ajoutons l'urgence demeure aussi temporellement délimitée, l'information demeure due postérieurement, sur les actes accomplis, leur raison et leurs conséquences, fastes ou péjoratives. La traçabilité de l'information dispensée, du consentement donné, mais encore des actes accomplis est un impératif absolu. L'article L4321-1 prévoit d'ailleurs qu' «Un compte rendu des actes accomplis dans ces conditions est remis au médecin dès son intervention ${ }^{106}$. Bien évidemment, le patient est aussi destinataire de cette information a posteriori.

Il convient enfin d'envisager la possibilité d'intervention du masseur-kinésithérapeute auprès d'un mineur [45] ou d'un majeur protégé ${ }^{107}$. Rappelons que l'article L1111-2 dispose que «Les intéressés ont le droit de recevoir eux-mêmes une information et de participer à la prise de décision les concernant, d'une manière adaptée soit à leur degré de maturité s'agissant des mineurs, soit à leurs facultés de discernement s'agissant des majeurs sous tutelle ». Pareillement, l'article L1111-4 précise que «Le consentement du mineur ou du majeur sous tutelle doit être systématiquement recherché s'il est apte à exprimer sa volonté et à participer à la décision. Dans le cas où le refus d'un traitement par la personne titulaire de l'autorité parentale ou par le tuteur risque d'entraîner des conséquences graves pour la santé du mineur ou du majeur sous tutelle, le médecin ${ }^{108}$ délivre les soins indispensables». L'article R4321-84, quant à lui, énonce que «Le masseurkinésithérapeute appelé à donner des soins à un mineur ou à un majeur protégé s'efforce de prévenir ses parents ${ }^{109}$ ou son représentant légal et d'obtenir leur consentement. En cas d'urgence, même si ceux-ci ne peuvent être joints, le masseur-kinésithérapeute donne les soins nécessaires. Si l'avis de l'intéressé peut être recueilli, le masseur-kinésithérapeute en tient compte dans toute la mesure du possible ». La lecture croisée des articles L4321-1 et R4321-84 permet d'envisager qu'en situation d'urgence le praticien délivre des soins nécessaires à un mineur ou un majeur protégé, nonobstant l'absence de consentement des parents ou du tuteur, qu'il a vainement tenté de joindre $^{110}$. Là encore la prudence s'impose et la mise en œuvre de l'habilitation ouverte par l'article L4321-1 de pratiquer les premiers soins sur un mineur ou un majeur protégé, sans le consentement parental ou de la personne chargée de la protection du majeur, ne saurait être envisagée qu'avec circonspection.

\footnotetext{
${ }^{105}$ Pour lesquelles l'assistance attendue ne semble pas nécessairement ancrée dans la pratique de «premiers actes de soins nécessaires en masso-kinésithérapie ».

${ }^{106}$ Même si le texte ne le précise pas, on doit considérer que le patient peut s'opposer à cette transmission. Le médecin destinataire doit aussi être celui choisi par le patient.

107 V. Code civil Art. 459 et 459-1.

${ }^{108}$ Seul le médecin est visé par le texte, cela exclut de jure les autres professionnels de santé.

109 Le pluriel est utilisé et semble exclure la qualification d'acte usuel de l'autorité parentale pour lequel le consentement du second parent peut être présumé [45].

${ }^{110}$ Le praticien veillera à se ménager la preuve de ces vaines tentatives.
} 


\section{Bibliographie :}

1. Terré F. Introduction générale au droit. Dalloz $§ n^{\circ} 24$.

2. Carbonaro R, Latruffe C. Quelle urgence en kinésithérapie ? Kinesither Rev 2017; 17: 26-30.

3. Jean S, Rocton R. De l'urgence à définir l'urgence en Kinésithérapie. Kinesither Rev 2017; 17 : 51-6.

4. Mathieu P. Échanges Courrier des lecteurs, Au sujet de l'article : Carbonaro R, Latruffe C. Quelle urgence en kinésithérapie ? Kinesither Rev 2017 ; 17 : 61-2.

5. Vialla VF. Salutat vos Lucas medicus carissimus. L'ambivalence des devoirs du médecin', in, Mélanges en l'honneur de Gérard Mémeteau. Droit médical et éthique médicale : regards contemporains, Bordeaux, LEH Édition, 2015, 209-26, Dir. J. Léonhard, B. Py, Vialla F.

6. Sicard D, et al. Penser solidairement la fin de vie - Commission de réflexion sur la fin de vie en France. Rapport remis à M. F. Hollande, Président de la République, p.33, https://www.ladocumentationfrancaise.fr/rapports-publics/124000675/index.shtml

7. Nicaise EV. Des instruments du chirurgien. In: de Chauliac G. La grande chirurgie. Chirurgien, maître en médecine de l'Université de Montpellier composée en l'an 1363 Paris, Alcan, 1890, p8.

8. Vialla F. La faute d'humanisme. in 10 ans d'application de la loi Kouchner, sous Dir. Bloch L, Combeau P, Groutel H. RGDM, n 17, 2013, 61-81.

9. Véron P. La décision médicale. Thèse Droit Montpellier 2015.

10. Pierron JP. Une nouvelle figure du patient ? Les transformations contemporaines de la relation de soins. Sciences sociales et santé, 2007, $\mathrm{n}^{\circ}$ 25-2, 43

11. Portes L. À la recherche d'une éthique médicale. Paris 1954, p163.

12. Vialla F. Consentir n'est pas imposer sa volonté. Revue Droit et Santé, n 80, 2017, 855-7 et Revue Droit et Santé n ${ }^{\circ} 86,2018,945-7$

13. Alt-Maes F. Les deux faces de l'information médicale : vers un nouvel équilibre des relations médecin-malade après la loi du 4 mars 2002 ? Gaz. Pal 2003 ; 350 : 3.

14. Chabas F. L'obligation médicale d'information en danger. JCP, G n 11, 15 Mars 2000, I 212.

15. Savatier R. Les Métamorphoses économiques et sociales du droit privé d'aujourd'hui. $2^{\text {ème }}$ série, Dalloz 1959, chap. VII sociologie juridique des professions libérales, p.189.

16. Binet JR. Ratification de la Convention d'Oviedo : la fin d'une longue attente, JCP G 2012, 89.

17. Mirkovic A. La ratification (enfin !) de la convention d'Oviedo sur les droits de l'homme et la biomédecine. D. 2012, 110-1.

18. Thierry D. La France enfin liée par la Convention d'Oviedo sur les droits de l'homme et la biomédecine. RDSS 2012. 839.

19. Millaire N. La Convention d'Oviedo est ratifiée ! Revue Droit et Santé, n 46, 2012, 272-4.

20. de Sola C, Erny I. La convention d'Oviedo sur les droits de l'homme et la biomédecine. in, Mélanges en l'honneur de Jean Michaud. Droit et bioéthique, Bordeaux, LEH Édition, 2012 (v. numérique 2013), 451-73.

21. Haute Autorité de Santé (HAS). Recommandation de bonne pratique, Délivrance de l'information à la personne sur son état de santé, mai 2012. https://www.hassante.fr/portail/upload/docs/application/pdf/2012-06/recommandations__delivrance_de_linformation_a_la_personne_sur_son_etat_de_sante.pdf 
22. St. Thomas d'Aquin. Commentaire sur les sentences de Pierre Lombard. IV, 30 question 1 ; Summa Theologiae Supplementum 1-68, quaestio 51.

23. Roland VH, Boyer L. Adages du droit français. Litec, n58, p106.

24. Girer M. Contribution à une analyse rénovée de la relation de soins. Essai de remise en cause du contrat médical. Bordeaux, LEH Édition, 2008 (v. numérique 2010), Collection Thèses et «La qualification juridique du consentement aux soins : accord contractuel, droit fondamental de la personne ? », in AFDS (Dir.), Consentement et santé, Paris, Dalloz, coll. 'Thèmes et commentaires », 2014, p.55.

25. Véron P, Vialla F. Qualification juridique du «colloque singulier» : faut-il en finir avec le contrat? Mélanges en l'honneur du Pr. Patrick Molinari, 2018 Thémis Montréal, avec Véron P, 245-77.

26. Malaurie P, Aynès L, Stoffel-Munck P. Les obligations, Defrénois, 2e éd. 2004, n 321 ; Véron P. La décision médicale, op. cit.

27. Serlooten P. Vers une responsabilité professionnelle. Mélanges Hébraud, 1981, 805, spéc. n 13.

28. Mathieu B. La bioéthique. Dalloz 2009, coll. Connaissance du droit, p.52.

29. Kelsen, Reine Rechtslehre 1934, Théorie pure du droit, trad. CH. Eisenmann, Paris Dalloz 1962.

30. Bruylant LGDJ 1999 ; Théorie générale des normes, Léviathan PUF 1996

31. Kelsen. Qu'est-ce que la théorie pure du droit? Droit et Société 1992 22, 551-68, https://www.persee.fr/doc/dreso_0769-3362_1992_num_22_1_1187

32. Garay A. Volontés et libertés dans la relation médecin-malade : la mise à l'épreuve des articles 16-3 du Code civil et L. 1111-4 du Code de la santé publique. RGDM, n 10, 2003, 151.

33. Byk Ch. Urgence et thérapie : rigueur et évolution du droit. RGDM, n 3, 2000.

34. CornuG . Droit civil. T. I, 12e éd. Domat-Montchrestien 2005, nº 417.

35. Direction Générale de la Santé. Circulaire DH.DGS-90.326 du 15 février 1990, §3.4.2 Consultations non programmées en établissement de santé.

36. Claudet I, Joly-Pedespan L. Consultations de routine aux urgences : faut-il gérer ou lutter ? Arch Pédiatrie 2008 ; 15 : 1733-8. Doi : 10.1016/j.arcped.2008.09.025.

37. Peneff J. Les malades des urgences, une forme de consommation médicale. Paris : Edition Métailié 2000.

38. Macron A. Circulaire relative à l'urgence en kinésithérapie : en matière de droit mou le CNOMK a la main dure... Rev. Droit et Santé 2017 ; 77: 387-90.

39. Chopard-Dit-Jean A. La responsabilité du médecin au service des urgences. Bordeaux, LEH Édition, 2011, Mémoires numériques de la BNDS.

40. Binet JR, Chopard-Dit-Jean A, Desmettre T, Guinchard Ch, Jacques-Jouvenot C, Philippe C. Approche multidisciplinaire de la vulnérabilité dans le contexte de l'urgence médicale. RGDM, $2011 ; 39: 157-80$.

41. Champlong L. Urgence et consentement en matière médicale. Rev Droit Santé 2012 ; 49: 58798.

42. Cornu G. Vocabulaire juridique. Association Henri CAPITANT, P.U.F.

43. Jestaz Ph. L'urgence et les principes classiques du droit civil. LGDJ (1968); Jean S, Rocton R. De l'urgence à définir l'urgence en Kinésithérapie. Kinesither Rev 2017; 17: 51-6. 
44. Rapport explicatif à la Convention sur les droits de l'homme et la biomédecine. DIR/JUR (97) I, Conseil de l'Europe Strasbourg, 1997, p15.

45. Vialla F. Relation de soin et minorité. Petites affiches, 20 mars 2015 n 57, 4. 